\title{
Integral Formulas for a Foliation with a Unit Normal Vector Field
}

\author{
Vladimir Rovenski (D)
}

check for

updates

Citation: Rovenski, V. Integral Formulas for a Foliation with a Unit Normal Vector Field. Mathematics 2021, 9, 1764. https://doi.org/ $10.3390 /$ math 9151764

Academic Editor: Ion Mihai

Received: 26 June 2021

Accepted: 22 July 2021

Published: 26 July 2021

Publisher's Note: MDPI stays neutral with regard to jurisdictional claims in published maps and institutional affiliations.

Copyright: (C) 2021 by the author. Licensee MDPI, Basel, Switzerland. This article is an open access article distributed under the terms and conditions of the Creative Commons Attribution (CC BY) license (https:// creativecommons.org/licenses/by/ $4.0 /)$.
Department of Mathematics, University of Haifa, Mount Carmel, Haifa 3498838, Israel; vrovenski@univ.haifa.ac.il
Abstract: In this article, we prove integral formulas for a Riemannian manifold equipped with a foliation $\mathcal{F}$ and a unit vector field $N$ orthogonal to $\mathcal{F}$, and generalize known integral formulas (due to Brito-Langevin-Rosenberg and Andrzejewski-Walczak) for foliations of codimension one. Our integral formulas involve Newton transformations of the shape operator of $\mathcal{F}$ with respect to $N$ and the curvature tensor of the induced connection on the distribution $\mathcal{D}=T \mathcal{F} \oplus \operatorname{span}(N)$, and this decomposition of $\mathcal{D}$ can be regarded as a codimension-one foliation of a sub-Riemannian manifold. We apply our formulas to foliated (sub-)Riemannian manifolds with restrictions on the curvature and extrinsic geometry of the foliation.

Keywords: Riemannian manifold; foliation; harmonic distribution; Newton transformation; shape operator; $r$-th mean curvature

MSC: 53C12; 53C17

\section{Introduction}

Foliations, which are defined as partitions of a manifold into collections of submanifolds of the same dimension, called leaves, appeared in the 1940s in the works of G. Reeb and $\mathrm{Ch}$. Ehresmann, culminating in the book [1]. Since then, the subject has enjoyed a rapid development, see e.g., [2]. Extrinsic geometry of foliations of Riemannian manifolds was developed in recent years, see surveys in [3-9], and has applications in differential geometry and analysis on real and complex manifolds. By extrinsic geometry we mean properties of foliations on Riemannian manifolds which can be expressed in terms of the second fundamental form of the leaves and its invariants (principal curvatures, scalar mean curvature, higher order mean curvatures $\sigma_{r}{ }^{\prime}$ s for $r>1$ and so on). G. Reeb also published a paper [10] on extrinsic geometry of foliations, in which he proved that the integral of the mean curvature of the leaves of any codimension-one foliation on any closed Riemannian manifold equals zero,

$$
\int_{M} \sigma_{1} d \operatorname{vol}_{g}=0
$$

thus, either the mean curvature of the leaves $\sigma_{1} \equiv 0$ or $\sigma_{1}(x) \sigma_{1}\left(x^{\prime}\right)<0$ for some points $x \neq x^{\prime}$.

Integral formulas in Riemannian geometry can be viewed as "conservation laws" of quantities when the metric changes. Integral formulas are now the centerpiece of extrinsic geometry of foliations and are useful in several geometric situations: characterizing foliations, whose leaves have a given geometric property; prescribing the higher mean curvatures of the leaves of a foliation; minimizing functionals such as volume defined for tensor fields on a foliated manifold. In [4], the Newton transformations $T_{r}\left(A_{N}\right)$ of the shape operator $A_{N}$ of the leaves (with a unit normal vector field $N$ ) were applied to a codimension-one foliated $(n+1)$-dimensional Riemannian manifold (with the curvature 
tensor $R$ ), and a series of integral formulas with total $r$-th mean curvatures for $r \in[0, n-2]$ was proved:

$$
\int_{M}\left((r+2) \sigma_{r+2}-\operatorname{tr}_{\mathcal{F}}\left(T_{r}\left(A_{N}\right) R(\cdot, N) N\right)-\left\langle\operatorname{div}_{\mathcal{F}} T_{r}\left(A_{N}\right), \nabla_{N} N\right\rangle\right) \mathrm{d} \operatorname{vol}_{g}=0 .
$$

These integral formulas were obtained by applying the Divergence Theorem to suitable vector fields. The next to (1) integral formula in the series (2) for $r=0$ and $n \geq 2$ is, see [11],

$$
\int_{M}\left(2 \sigma_{2}-\operatorname{Ric}_{N, N}\right) \mathrm{d} \operatorname{vol}_{g}=0
$$

By (3), there are no totally umbilical codimension-one foliations of a closed manifold of negative Ricci curvature, and there are no harmonic codimension-one foliations of a closed manifold of positive Ricci curvature. It was proved in [6], and can be deduced from (2), that on a compact space form $M^{n+1}(c)$ the total $\sigma_{r}$ 's are independent of $\mathcal{F}$ :

$$
\int_{M} \sigma_{r} \mathrm{~d} \mathrm{vol}_{g}=\left\{\begin{array}{cc}
c^{r / 2}\left(\begin{array}{c}
n / 2 \\
r / 2
\end{array}\right) \operatorname{Vol}(M, g), & n \text { and } r \text { even, } \\
0, & \text { either } n \text { or } r \text { odd } .
\end{array}\right.
$$

The natural question arises: can we find integral formulas similar to (2) and (4) for foliations of arbitrary codimension? In studying this question, we consider a foliation $\mathcal{F}$ of any codimension, whose normal bundle has zero Euler class; thus, $\mathcal{F}$ can be equipped with a unit normal vector field $N$. Let

$$
\mathcal{D}=T \mathcal{F} \oplus \operatorname{span}(N)
$$

be a distribution on $M$ (subbundle of the tangent bundle $T M$ ) spanned by $T \mathcal{F}$ (the tangent bundle of $\mathcal{F}$ ) and $N$. In the article, we prove integral formulas (in Theorems 1-3 and Corollaries 3 and 4), which generalize (2) and (4) for codimension-one foliations. Our integral formulas involve $r$-th mean curvatures of $\mathcal{F}$ with respect to $N$, i.e., symmetric functions of the shape operator $A_{N}$ of $\mathcal{F}$, Newton transformations of $A_{N}$ and the curvature tensor of the induced connection on $\mathcal{D}$. We apply our formulas to foliations with restrictions on the curvature and the extrinsic geometry.

Please note that $(M, \mathcal{D})$ with the metric on $\mathcal{D}$ (e.g., the restriction of $g$ ) is the object of sub-Riemannian geometry, see [5]. Apparently, a foliated sub-Riemannian manifold $(M, \mathcal{D}, \mathcal{F}, g)$, i.e., the tangent bundle $T \mathcal{F}$ of a foliation $\mathcal{F}$ is a subbundle of $\mathcal{D}$, is a new (or little-studied) geometrical object. Therefore, the results (in Sections 3 and 4) can be interpreted as integral formulas for codimension-one foliations of a sub-Riemannian manifold.

\section{Preliminaries}

Here, using the induced linear connection on a distribution, we define the shape operator (with its Newton transformations) and the curvature tensor related to a codimension-one foliated sub-Riemannian manifold, then we prove three auxiliary lemmas.

Let $\mathcal{D}$ be an $(n+1)$-dimensional distribution on a smooth $m$-dimensional manifold $M$, i.e., a subbundle of $T M$ of rank $n+1$ (where $0<n<m$ ). In other words, to each point $x \in M$ we assign an $(n+1)$-dimensional subspace $\mathcal{D}_{x}$ of the tangent space $T_{x} M$ smoothly depending on $x$. A pair $(M, \mathcal{D})$, where $M$ is a manifold and $\mathcal{D}$ is a non-integrable distribution on $M$, is called a non-holonomic manifold, see [5]. The concept of a non-holonomic manifold was introduced for a geometric interpretation of constrained systems in classical mechanics. A sub-Riemannian manifold is a non-holonomic manifold $(M, \mathcal{D})$, equipped with a sub-Riemannian metric $g=\langle\cdot, \cdot\rangle$, i.e., the scalar product $g: \mathcal{D}_{x} \times \mathcal{D}_{x} \rightarrow \mathbb{R}$ for all $x \in M$, see [5]. Usually, they assume that the sub-Riemannian metric on the horizontal bundle $\mathcal{D}$ is extended to a Riemannian metric (also denoted by $g$ ) on the whole manifold $M$. This allows us to define the orthogonal distribution $\mathcal{D}^{\perp}$ (the vertical subbundle) such that $T M=\mathcal{D} \oplus \mathcal{D}^{\perp}$. 
The orthoprojector $P: T M \rightarrow \mathcal{D}$ onto the distribution $\mathcal{D}$ is characterized by the properties

$$
P=P^{*} \text { (self-adjoint), } \quad P^{2}=P,
$$

e.g., [12]. A sub-Riemannian manifold equipped with a foliation $\mathcal{F}$ such that the tangent bundle $T \mathcal{F}$ is a subbundle of the horizontal bundle will be called a foliated sub-Riemannian manifold. By Frobenius Theorem, e.g., ([5] Theorem 1.7), a foliation is determined by an involutive distribution, i.e., the Lie bracket of any two its vector fields also belongs to this distribution.

In this article, we assume that $\mathcal{F}$ is a codimension-one foliation relative to $\mathcal{D}$, i.e., $\operatorname{dim} \mathcal{F}=n$, and there exists a unit vector field $N$ orthogonal to $\mathcal{F}$ and tangent to $\mathcal{D}$. This means that the Euler characteristic of the subbundle $\mathcal{D}$ is zero and the following orthogonal decomposition is valid, see (5):

$$
\mathcal{D}=T \mathcal{F} \oplus \operatorname{span}(N) .
$$

The Levi-Civita connection $\nabla$ on $(M, g)$ induces a linear connection $\nabla^{P}$ on the distribution $\mathcal{D}=P(T M)$ :

$$
\nabla_{X}^{P} P Y=P \nabla_{X} P Y, \quad X, Y \in \Gamma(T M),
$$

which is compatible with the metric: $X\langle U, V\rangle=\left\langle\nabla_{X}^{P} U, V\right\rangle+\left\langle U, \nabla_{X}^{P} V\right\rangle$ for any sections $U$ and $V$ of $\mathcal{D}$ and $X \in \Gamma(T M)$. Define the horizontal vector field

$$
\mathcal{Z}=\nabla_{N}^{P} N
$$

and note that $\nabla_{N} N$ is the curvature vector of $N$-curves in $(M, g)$.

The shape operator $A_{N}: T \mathcal{F} \rightarrow T \mathcal{F}$ of the foliation $\mathcal{F}$ with respect to $N$ is defined by

$$
A_{N}(X)=-\nabla_{X}^{P} N, \quad X \in T \mathcal{F} .
$$

The elementary symmetric functions $\sigma_{j}\left(A_{N}\right)$ of $A_{N}$ (called the $r$-th mean curvatures of $\mathcal{F}$ with respect to $N$ in $\mathcal{D}$ ) are given by the equality

$$
\sum_{r=0}^{n} \sigma_{r}\left(A_{N}\right) t^{r}=\operatorname{det}\left(\operatorname{id}_{T \mathcal{F}}+t A_{N}\right), \quad t \in \mathbb{R} .
$$

Note that $\sigma_{r}\left(A_{N}\right)=\sum_{i_{1}<\cdots<i_{r}} \lambda_{i_{1}} \cdots \lambda_{i_{r}}$, where $\lambda_{1} \leq \ldots \leq \lambda_{n}$ are the eigenvalues of $A_{N}$. Let $\tau_{j}\left(A_{N}\right)=\operatorname{tr} A_{N}^{j}$ for $j \in \mathbb{N}$ be the power sums symmetric functions of $A_{N}$. For example, $\sigma_{0}\left(A_{N}\right)=1, \sigma_{1}\left(A_{N}\right)=\tau_{1}\left(A_{N}\right)=\operatorname{tr} A_{N}, \sigma_{n}\left(A_{N}\right)=\operatorname{det} A_{N}$, and

$$
2 \sigma_{2}\left(A_{N}\right)=\tau_{1}^{2}\left(A_{N}\right)-\tau_{2}\left(A_{N}\right)
$$

For short, we set

$$
\sigma_{r}=\sigma_{r}\left(A_{N}\right), \quad \tau_{r}=\tau_{r}\left(A_{N}\right) .
$$

Next, we introduce the curvature tensor $R^{P}: T M \times T M \rightarrow \operatorname{End}(\mathcal{D})$ of the connection $\nabla^{P}$ :

$$
R^{P}(X, Y)=\nabla_{X}^{P} \nabla_{Y}^{P}-\nabla_{Y}^{P} \nabla_{X}^{P}-\nabla_{[X, Y]}^{P} .
$$

Set $R^{P}(X, Y, V, U)=\left\langle R^{P}(X, Y) V, U\right\rangle$ for $U, V \in \mathcal{D}$. The sectional $P$-curvature of a plane spanned by non-collinear vectors $X, Y$ is $R^{P}(X, Y, Y, X) /\left(X^{2} Y^{2}-\langle X, Y\rangle^{2}\right)$.

Obviously, $R^{P}(Y, X)=-R^{P}(X, Y)$ (for any linear connection). Since $\nabla^{P}$ is compatible with the metric, then the anti-symmetry for the last pair of vectors is valid, e.g., [13],

$$
R^{P}(X, Y, V, U)=-R^{P}(X, Y, U, V) .
$$

Lemma 1. For $\mathcal{D}=T \mathcal{F} \oplus \operatorname{span}(N)$ on $(M, g)$, the following Codazzi type equation is valid:

$$
\left(\nabla_{X}^{\mathcal{F}} A_{N}\right) Y-\left(\nabla_{Y}^{\mathcal{F}} A_{N}\right) X=-R^{P}(X, Y) N, \quad X, Y \in T \mathcal{F} .
$$


Proof. Recall the Codazzi's equation for a foliation (or a submanifold) of $(M, g)$ :

$$
\left(\nabla_{X} h\right)(Y, U)-\left(\nabla_{Y} h\right)(X, U)=(R(X, Y) U)^{\perp},
$$

where $R: T M \times T M \rightarrow \operatorname{End}(T M)$ is the curvature tensor of the Levi-Civita connection,

$$
R(X, Y)=\nabla_{X} \nabla_{Y}-\nabla_{Y} \nabla_{X}-\nabla_{[X, Y]}
$$

${ }^{\perp}$ denotes the projection onto the vector bundle orthogonal to $\mathcal{F}$, and $h: T \mathcal{F} \times T \mathcal{F} \rightarrow$ $(T \mathcal{F})^{\perp}$ is the second fundamental form of $\mathcal{F}$ in $(M, g)$ defined by

$$
h(X, Y)=\left(\nabla_{X} Y\right)^{\perp} .
$$

From (11), for all vectors $X, Y, U \in T \mathcal{F}$ we obtain

$$
\begin{aligned}
& \left\langle\left(\nabla_{X}^{\mathcal{F}} A_{N}\right) Y-\left(\nabla_{Y}^{\mathcal{F}} A_{N}\right) X, U\right\rangle+\left\langle h(X, U), \nabla_{Y} N\right\rangle \\
& -\left\langle h(Y, U), \nabla_{X} N\right\rangle=-\langle R(X, Y) N, U\rangle .
\end{aligned}
$$

Applying the orthoprojector on the vector bundle orthogonal to $\mathcal{F}$, we find

$$
\begin{aligned}
& \langle R(X, Y) N, U\rangle=\left\langle R^{P}(X, Y) N, U\right\rangle+\left\langle\nabla_{X}\left(\left(\nabla_{Y} N\right)^{\perp}\right)\right. \\
& \left.-\nabla_{Y}\left(\left(\nabla_{X} N\right)^{\perp}\right)-\nabla_{[X, Y]^{\perp}} N, U\right\rangle .
\end{aligned}
$$

Using the equalities $[X, Y]^{\perp}=0$ (since $T \mathcal{F}$ is integrable), (13) and

$$
\begin{aligned}
\left\langle h(X, U), \nabla_{Y} N\right\rangle & =\left\langle\nabla_{Y} N,\left(\nabla_{X} U\right)^{\perp}\right\rangle=-\left\langle\nabla_{X}\left(\left(\nabla_{Y} N\right)^{\perp}\right), U\right\rangle, \\
\left\langle h(Y, U), \nabla_{X} N\right\rangle & =\left\langle\nabla_{X} N,\left(\nabla_{Y} U\right)^{\perp}\right\rangle=-\left\langle\nabla_{Y}\left(\left(\nabla_{X} N\right)^{\perp}\right), U\right\rangle,
\end{aligned}
$$

in (12) completes the proof.

The following lemma generalizes [4] (Lemma 3.1).

Lemma 2. Let $\left\{e_{i}\right\}$ be a local orthonormal frame of $T \mathcal{F}$ such that at a point $x \in M$ :

- $\quad \nabla_{X}^{\mathcal{F}} e_{i}=0(1 \leq i \leq n)$ for any vector $X \in T_{x} M$;

- $\quad \nabla_{\xi}^{P} e_{i}=0(1 \leq i \leq n)$ for any vector $\xi \in \mathcal{D}_{x}^{\perp} M$.

Then the following equality is valid at $x \in M$ :

$$
\begin{aligned}
& \left\langle\nabla_{e_{i}} \mathcal{Z}, e_{j}\right\rangle=\left\langle A_{N}^{2} e_{i}, e_{j}\right\rangle+\left\langle R^{P}\left(e_{i}, N\right) N, e_{j}\right\rangle \\
& -\left\langle\left(\nabla_{N}^{\mathcal{F}} A_{N}\right) e_{i}, e_{j}\right\rangle+\left\langle\mathcal{Z}, e_{i}\right\rangle\left\langle\mathcal{Z}, e_{j}\right\rangle .
\end{aligned}
$$

Proof. Taking covariant derivative of $\left\langle\mathcal{Z}, e_{j}\right\rangle=-\left\langle N, \nabla_{N} e_{j}\right\rangle$ with respect to $e_{i}$, we find

$$
-\left\langle\mathcal{Z}, \nabla_{e_{i}} e_{j}\right\rangle=\left\langle\nabla_{e_{i}} \mathcal{Z}, e_{j}\right\rangle+\left\langle\nabla_{e_{i}} N, P \nabla_{N} e_{j}\right\rangle+\left\langle N, \nabla_{e_{i}} P \nabla_{N} e_{j}\right\rangle .
$$

For a foliation $\mathcal{F}$, we obtain

$$
\left\langle\left(\nabla_{N}^{\mathcal{F}} A_{N}\right) e_{i}, e_{j}\right\rangle=\nabla_{N}\left\langle N, \nabla_{e_{i}} e_{j}\right\rangle=\left\langle\mathcal{Z}, \nabla_{e_{i}} e_{j}\right\rangle+\left\langle N, \nabla_{N} P \nabla_{e_{i}} e_{j}\right\rangle
$$

Therefore, using (8), we calculate at the point $x \in M$ :

$$
\begin{aligned}
& \left\langle A_{N}^{2} e_{i}, e_{j}\right\rangle+\left\langle R^{P}\left(e_{i}, N\right) N, e_{j}\right\rangle-\left\langle\left(\nabla_{N}^{\mathcal{F}} A_{N}\right) e_{i}, e_{j}\right\rangle \\
& =\left\langle A_{N}^{2} e_{i}, e_{j}\right\rangle-\left\langle R^{P}\left(e_{i}, N\right) e_{j}, N\right\rangle+N\left\langle\nabla_{e_{i}} N, e_{j}\right\rangle \\
& =\left\langle A_{N}^{2} e_{i}, e_{j}\right\rangle-\left\langle\mathcal{Z}, \nabla_{e_{i}} e_{j}\right\rangle-\left\langle\nabla_{e_{i}} P \nabla_{N} e_{j}, N\right\rangle+\left\langle\nabla_{\left[e_{i}, N\right]} e_{j}, N\right\rangle .
\end{aligned}
$$


By conditions at $x \in M$, we rewrite the last term in (16) as

$$
\left\langle\nabla_{P\left[e_{i}, N\right]} e_{j}, N\right\rangle=\left\langle\nabla_{\left[e_{i}, N\right]} e_{j}, N\right\rangle .
$$

Then, using (15) and the following equalities at $x \in M$ :

$$
\begin{aligned}
& P \nabla_{e_{i}} N=\sum_{1 \leq j \leq n}\left\langle\nabla_{e_{i}} N, e_{j}\right\rangle e_{j}, \quad P \nabla_{N} e_{i}=\left\langle\nabla_{N} e_{i}, N\right\rangle N, \\
& \left\langle A_{N}^{2} e_{i}, e_{j}\right\rangle=\left\langle\nabla_{e_{i}} N, N\right\rangle\left\langle\nabla_{N} e_{j}, N\right\rangle,
\end{aligned}
$$

we simplify the last line in (16) as

$$
\left\langle\nabla_{e_{i}} \mathcal{Z}, e_{j}\right\rangle-\left\langle\mathcal{Z}, e_{i}\right\rangle\left\langle\mathcal{Z}, e_{j}\right\rangle
$$

From the above, the claim follows.

Many authors investigated $r$-th mean curvatures of foliations and hypersurfaces of Riemannian manifolds using the Newton transformations of the shape operator, see [4].

Definition 1. The Newton transformations $T_{r}\left(A_{N}\right)$ of the shape operator $A_{N}$ of an n-dimensional foliation $\mathcal{F}$ of a sub-Riemannian manifold $(M, \mathcal{D}, g)$ are defined recursively or explicitly by

$$
\begin{gathered}
T_{0}\left(A_{N}\right)=\operatorname{id}_{T \mathcal{F}}, \quad T_{r}\left(A_{N}\right)=\sigma_{r} \operatorname{id}_{T \mathcal{F}}-A_{N} T_{r-1}\left(A_{N}\right), \quad 1 \leq r \leq n, \\
T_{r}\left(A_{N}\right)=\sum_{j=0}^{r}(-1)^{j} \sigma_{r-j} A_{N}^{j}=\sigma_{r} \operatorname{id}_{T \mathcal{F}}-\sigma_{r-1} A_{N}+\ldots+(-1)^{r} A_{N}^{r} .
\end{gathered}
$$

For example, $T_{1}\left(A_{N}\right)=\sigma_{1} \operatorname{id}_{T \mathcal{F}}-A_{N}$ and $T_{n}\left(A_{N}\right)=0$. Notice that $A_{N}$ and $T_{r}\left(A_{N}\right)$ commute. The following properties of $T_{r}\left(A_{N}\right)$ are proved similarly as for codimension-one foliations of a Riemannian manifold, e.g., [4] (or [7] Lemma 1.3).

Lemma 3. For the shape operator $A_{N}$ we have

$$
\begin{aligned}
\operatorname{tr}_{\mathcal{F}} T_{r}\left(A_{N}\right) & =(n-r) \sigma_{r} \\
\operatorname{tr}_{\mathcal{F}}\left(A_{N} \cdot T_{r}\left(A_{N}\right)\right) & =(r+1) \sigma_{r+1} \\
\operatorname{tr}_{\mathcal{F}}\left(A_{N}^{2} \cdot T_{r}\left(A_{N}\right)\right) & =\sigma_{1} \sigma_{r+1}-(r+2) \sigma_{r+2}, \\
\operatorname{tr}_{\mathcal{F}}\left(T_{r-1}\left(A_{N}\right)\left(\nabla_{X}^{\mathcal{F}} A_{N}\right)\right) & =X\left(\sigma_{r}\right), \quad X \in T \mathcal{F}, \quad r>0 .
\end{aligned}
$$

On the other hand, since the $(1,1)$-tensors $A_{N}$ and $T_{r}\left(A_{N}\right)$ are self-adjoint, we have

$$
\left\langle\left(\nabla_{X}^{\mathcal{F}} T_{r}\left(A_{N}\right)\right) Y, V\right\rangle=\left\langle\left(\nabla_{X}^{\mathcal{F}} T_{r}\left(A_{N}\right)\right) V, Y\right\rangle, \quad X, Y, V \in T \mathcal{F} .
$$

\section{Main Results}

Here, we prove a series of integral formulas for a codimension-one foliated subRiemannian manifold $(M, \mathcal{D}, \mathcal{F}, g)$ with $\mathcal{D}=T \mathcal{F} \oplus \operatorname{span}(N)$.

Recall that the $\mathcal{F}$-divergence of a vector field $X$ tangent to $\mathcal{F}$ on $(M, g)$ is defined by

$$
\operatorname{div} X=\sum_{i=1}^{n}\left\langle\nabla_{e_{i}} X, e_{i}\right\rangle,
$$

where $\left\{e_{i}\right\}$ is a local orthonormal frame of $T \mathcal{F}$. Following [4], define the $\mathcal{F}$-divergence of the Newton transformation $T_{r}\left(A_{N}\right)$ by

$$
\operatorname{div}_{\mathcal{F}} T_{r}\left(A_{N}\right)=\sum_{i=1}^{n}\left(\nabla_{e_{i}}^{\mathcal{F}} T_{r}\left(A_{N}\right)\right) e_{i} .
$$

Please note that $\operatorname{div}_{\mathcal{F}} T_{0}\left(A_{N}\right)=0$.

For any $X \in \mathcal{D}$, define a linear operator $\mathcal{R}_{X}^{P}: T \mathcal{F} \rightarrow T \mathcal{F}$ by

$$
\mathcal{R}_{X}^{P}: V \rightarrow R^{P}(V, X) N, \quad V \in T \mathcal{F} .
$$


The following result generalizes Lemma 2.2 in [4].

Lemma 4. The leafwise divergence of $T_{r}\left(A_{N}\right)$ for $r>0$ satisfies the inductive formula

$$
\left\langle\operatorname{div}_{\mathcal{F}} T_{r}\left(A_{N}\right), X\right\rangle=-\left\langle\operatorname{div}_{\mathcal{F}} T_{r-1}\left(A_{N}\right), A_{N} X\right\rangle+\operatorname{tr}_{\mathcal{F}}\left(T_{r-1}\left(A_{N}\right) \mathcal{R}_{X}^{P}\right),
$$

where $X \in \Gamma(\mathcal{F})$. Equivalently, for $r>0$ we have

$$
\left\langle\operatorname{div}_{\mathcal{F}} T_{r}\left(A_{N}\right), X\right\rangle=\sum_{j=1}^{r}(-1)^{j-1} \operatorname{tr}_{\mathcal{F}}\left(T_{r-j}\left(A_{N}\right) \mathcal{R}_{A_{N}^{j-1} X}^{P}\right) .
$$

Proof. Using the recursive definition of $T_{r}\left(A_{N}\right)$, we have

$$
\operatorname{div}_{\mathcal{F}} T_{r}\left(A_{N}\right)=\nabla^{\mathcal{F}} \sigma_{r}-A_{N} \operatorname{div}_{\mathcal{F}} T_{r-1}\left(A_{N}\right)-\sum_{i=1}^{n}\left(\nabla_{e_{i}}^{\mathcal{F}} A_{N}\right) T_{r-1}\left(A_{N}\right) e_{i}
$$

Applying Codazzi type equation (10) and the last formula in Lemma 3, we obtain

$$
\begin{aligned}
& \sum_{i=1}^{n}\left\langle\left(\nabla_{e_{i}}^{\mathcal{F}} A_{N}\right) T_{r-1}\left(A_{N}\right) e_{i}, X\right\rangle=\sum_{i=1}^{n}\left\langle T_{r-1}\left(A_{N}\right) e_{i},\left(\nabla_{e_{i}}^{\mathcal{F}} A_{N}\right) X\right\rangle \\
& =\sum_{i=1}^{n}\left\langle T_{r-1}\left(A_{N}\right) e_{i},\left(\nabla_{X}^{\mathcal{F}} A_{N}\right) e_{i}-R^{P}\left(e_{i}, X\right) N\right\rangle \\
& =\operatorname{tr}_{\mathcal{F}}\left(T_{r-1}\left(A_{N}\right)\left(\nabla_{X}^{\mathcal{F}} A_{N}\right)\right)-\sum_{i=1}^{n}\left\langle R^{P}\left(e_{i}, X\right) N, T_{r-1}\left(A_{N}\right) e_{i}\right\rangle \\
& =X\left(\sigma_{r}\right)-\operatorname{tr}_{\mathcal{F}}\left(T_{r-1}\left(A_{N}\right) \mathcal{R}_{X}^{P}\right) .
\end{aligned}
$$

Here, we used $R^{P}\left(e_{i}, X\right) N=\mathcal{R}_{X}^{P}\left(e_{i}\right)$, see (19). Hence, the inductive Formula (20) holds. Finally, (21) follows directly from the above.

Remark 1. (a) If $P=\mathrm{id}_{T M}$, i.e., $\mathcal{F}$ is a codimension-one foliation of $M$, then $R^{P}=R$, and using the symmetry $\langle R(X, Y) U, V\rangle=\langle R(U, V) X, Y\rangle$, we simplify Equation (20) to the form

$$
\operatorname{div}_{\mathcal{F}} T_{r}\left(A_{N}\right)=-A_{N} \operatorname{div}_{\mathcal{F}} T_{r-1}\left(A_{N}\right)+\sum_{i=1}^{n}\left(R\left(N, T_{r-1}\left(A_{N}\right) e_{i}\right) e_{i}\right)^{\top},
$$

(see Lemma 2.2 in [4]), where ${ }^{\top}$ denotes the orthogonal projection on the vector bundle $T \mathcal{F}$.

(b) Let the distribution $T \mathcal{F}$ be P-curvature invariant, i.e.,

$$
R^{P}(X, Y) V \in T \mathcal{F}, \quad X, Y, V \in T \mathcal{F}
$$

By (9), Equation (21) implies that $\operatorname{div}_{\mathcal{F}} T_{r}\left(A_{N}\right)=0$ for every $r \geq 0$. Condition (22) is obviously satisfied, if the distribution $T \mathcal{F}$ is auto-parallel, i.e., $\nabla_{X} Y \in \Gamma(T \mathcal{F})$ for all $X, Y \in \Gamma(T \mathcal{F})$. A sufficient condition for (22) is the constancy of the sectional P-curvature, i.e., the following equality with some real constant $c$ :

$$
R^{P}(X, Y) V=c(\langle Y, V\rangle X-\langle X, V\rangle Y), \quad X, Y, V \in \mathcal{D}
$$

The following result generalizes Proposition 3.3 in [4].

Theorem 1. For any compact leaf $L$ of $\mathcal{F}$ we have

$$
\begin{aligned}
& \int_{L}\left((r+2) \sigma_{r+2}+N\left(\sigma_{r+1}\right)-\sigma_{1} \sigma_{r+1}-\operatorname{tr}_{\mathcal{F}}\left(T_{r}\left(A_{N}\right) \mathcal{R}_{N}^{P}\right)\right. \\
& \left.\quad-\left\langle T_{r}\left(A_{N}\right) \mathcal{Z}, \mathcal{Z}\right\rangle-\underline{\left\langle\operatorname{div}_{\mathcal{F}} T_{r}\left(A_{N}\right), \mathcal{Z}\right\rangle}\right) \mathrm{d} \mathrm{vol}_{L}=0, \quad r \in[0, n-2],
\end{aligned}
$$

where the underlined term is given by (21) with $X=\mathcal{Z}$. 
Proof. Using (17) and (18), we compute the divergence of the vector field $T_{r}\left(A_{N}\right) \mathcal{Z}$ as follows:

$$
\begin{aligned}
\operatorname{div}_{\mathcal{F}} T_{r}\left(A_{N}\right) \mathcal{Z} & =\sum_{i=1}^{n}\left\langle\nabla_{e_{i}}\left(T_{r}\left(A_{N}\right) \mathcal{Z}\right), e_{i}\right\rangle \\
& =\left\langle\operatorname{div}_{\mathcal{F}} T_{r}\left(A_{N}\right), \mathcal{Z}\right\rangle+\sum_{i=1}^{n}\left\langle\nabla_{e_{i}} \mathcal{Z}, T_{r}\left(A_{N}\right) e_{i}\right\rangle .
\end{aligned}
$$

Using (14) and (19), we compute $\sum_{i=1}^{n}\left\langle\nabla_{e_{i}} \mathcal{Z}, T_{r}\left(A_{N}\right) e_{i}\right\rangle$ as

$$
\begin{aligned}
& \sum_{i=1}^{n}\left(\left\langle A_{N}^{2} e_{i}+R^{P}\left(e_{i}, N\right) N-\left(\nabla_{N}^{\mathcal{F}} A_{N}\right) e_{i}, T_{r}\left(A_{N}\right) e_{i}\right\rangle+\left\langle\mathcal{Z}, e_{i}\right\rangle\left\langle\mathcal{Z}, T_{r}\left(A_{N}\right) e_{i}\right\rangle\right) \\
& \quad=-\operatorname{tr}_{\mathcal{F}}\left(T_{r}\left(A_{N}\right)\left(\nabla_{N}^{\mathcal{F}} A_{N}-A_{N}^{2}-\mathcal{R}_{N}^{P}\right)\right)+\left\langle T_{r}\left(A_{N}\right) \mathcal{Z}, \mathcal{Z}\right\rangle .
\end{aligned}
$$

By Lemma 3, we can write

$$
\operatorname{tr}_{\mathcal{F}}\left(T_{r}\left(A_{N}\right)\left(\nabla_{N}^{\mathcal{F}} A_{N}-A_{N}^{2}-\mathcal{R}_{N}^{P}\right)\right)=N\left(\sigma_{r+1}\right)-\sigma_{1} \sigma_{r+1}+(r+2) \sigma_{r+2}-\operatorname{tr}_{\mathcal{F}}\left(T_{r}\left(A_{N}\right) \mathcal{R}_{N}^{P}\right) .
$$

Finally, we obtain

$$
\begin{aligned}
\sum_{i=1}^{n}\left\langle\nabla_{e_{i}} \mathcal{Z}, T_{r}\left(A_{N}\right) e_{i}\right\rangle= & -N\left(\sigma_{r+1}\right)+\sigma_{1} \sigma_{r+1}-(r+2) \sigma_{r+2} \\
& +\left\langle T_{r}\left(A_{N}\right) \mathcal{Z}, \mathcal{Z}\right\rangle+\operatorname{tr}_{\mathcal{F}}\left(T_{r}\left(A_{N}\right) \mathcal{R}_{N}^{P}\right) .
\end{aligned}
$$

This proves the following:

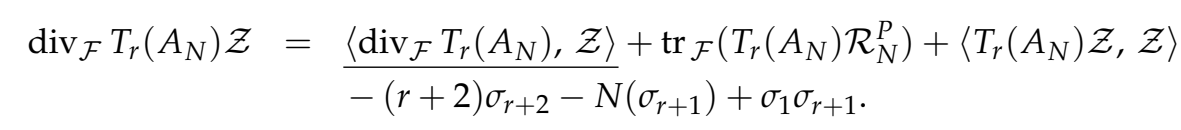

Applying the Divergence Theorem to (25) along any compact leaf, we obtain (24).

We can view $\operatorname{tr}_{\mathcal{F}} \mathcal{R}_{X}^{P}=\sum_{i=1}^{n}\left\langle R^{P}\left(e_{i}, X\right) N, e_{i}\right\rangle$ as the Ricci P-curvature $\operatorname{Ric}_{X, N}^{P}$. Then

$$
\operatorname{Ric}_{N, N}^{P}=\operatorname{tr}_{\mathcal{F}} \mathcal{R}_{N}^{P}
$$

is the Ricci $P$-curvature in the $N$-direction.

Corollary 1. Let $\tau_{1}=$ const. and $\operatorname{Ric}_{N, N}^{P}>0$. Then $\mathcal{F}$ has no compact leaves.

Proof. Let $L$ be a compact leaf. By (24) with $r=0$, using $2 \sigma_{2}=\tau_{1}^{2}-\tau_{2}$, we obtain

$$
\int_{L}\left(\tau_{2}-N\left(\tau_{1}\right)+\operatorname{tr}_{\mathcal{F}} \mathcal{R}_{N}^{P}+\langle\mathcal{Z}, \mathcal{Z}\rangle\right) \mathrm{d}^{v l_{L}}=0 .
$$

By conditions and (26), we obtain $N\left(\tau_{1}\right)=0$ and $\operatorname{tr}_{\mathcal{F}} \mathcal{R}_{N}^{P}>0$. This yields a contradiction.

For any vector field $X$ in $T \mathcal{F}$, we have

$$
\operatorname{div} X=\operatorname{div}_{\mathcal{F}} X-\langle X, \mathcal{Z}\rangle-\left\langle X, H^{\perp}\right\rangle,
$$

where $H^{\perp}$ is the mean curvature vector field of the distribution $\mathcal{D}^{\perp}$ (orthogonal to $\mathcal{D}$ ) in $(M, g)$. Recall that a distribution on a Riemannian manifold is called harmonic if its mean curvature vector field vanishes. There are topological restrictions for the existence of a Riemannian metric on closed manifold, for which a given distribution becomes harmonic, see [14].

The following statement generalizes (1). 
Theorem 2. For a closed sub-Riemannian manifold $(M, \mathcal{D}, g)$ with $\mathcal{D}=T \mathcal{F} \oplus \operatorname{span}(N)$ and a harmonic distribution $\mathcal{D}^{\perp}$, the following integral formula is valid:

$$
\int_{M} \sigma_{1} \mathrm{~d} \operatorname{vol}_{g}=0
$$

Proof. Recall that $\sigma_{1}=\operatorname{tr} A_{N}$, see (6), and observe that

$$
\operatorname{div}_{\mathcal{F}} N=\sum_{i=1}^{n}\left\langle\nabla_{e_{i}} N, e_{i}\right\rangle=-\sigma_{1} .
$$

Thus, using the assumption $H^{\perp}=0$, we obtain

$$
\operatorname{div} N=\operatorname{div}_{\mathcal{F}} N-\left\langle N, H^{\perp}\right\rangle=-\sigma_{1} .
$$

Applying the Divergence Theorem, yields (28).

We supplement (28) with a series of integral formulas with total $r$-th mean curvatures for $r \in[0, n-2]$, which generalize (2).

Theorem 3. For a closed sub-Riemannian manifold $(M, g)$ with $\mathcal{D}=T \mathcal{F} \oplus \operatorname{span}(N)$ and a harmonic distribution $\mathcal{D}^{\perp}$, the following integral formula is valid for $r \in[0, n-2]$ :

$$
\int_{M}\left((r+2) \sigma_{r+2}-\operatorname{tr}_{\mathcal{F}}\left(T_{r}\left(A_{N}\right) \mathcal{R}_{N}^{P}\right)-\underline{\left\langle\operatorname{div}_{\mathcal{F}} T_{r}\left(A_{N}\right), \mathcal{Z}\right\rangle}\right) \mathrm{d} \operatorname{vol}_{g}=0,
$$

where the underlined term is given by (21) with $X=\mathcal{Z}$.

Proof. Using (29), we calculate the divergence of $\sigma_{r+1} \cdot N$ as

$$
\operatorname{div}\left(\sigma_{r+1} \cdot N\right)=\sigma_{r+1} \operatorname{div} N+N\left(\sigma_{r+1}\right)=N\left(\sigma_{r+1}\right)-\sigma_{1} \sigma_{r+1} .
$$

Then, by (27) with $X=T_{r}\left(A_{N}\right) \mathcal{Z}$, (since $\mathcal{D}^{\perp}$ is a harmonic distribution), we obtain

$$
\operatorname{div}\left(T_{r}\left(A_{N}\right) \mathcal{Z}+\sigma_{r+1} N\right)=\left\langle\operatorname{div}_{\mathcal{F}} T_{r}\left(A_{N}\right), \mathcal{Z}\right\rangle-(r+2) \sigma_{r+2}+\operatorname{tr}_{\mathcal{F}}\left(T_{r}\left(A_{N}\right) \mathcal{R}_{N}^{P}\right) .
$$

Applying the Divergence Theorem, we obtain (30).

Example 1. If $N$ is a P-geodesic vector field, i.e., $\mathcal{Z}=0$, then (30) shortens to the formula

$$
\int_{M}\left((r+2) \sigma_{r+2}-\operatorname{tr}_{\mathcal{F}}\left(T_{r}\left(A_{N}\right) \mathcal{R}_{N}^{P}\right)\right) \mathrm{d} \operatorname{vol}_{g}=0 .
$$

For $r=0$, (30) gives us the following generalization of (3):

$$
\int_{M}\left(2 \sigma_{2}-\operatorname{Ric}_{N, N}^{P}\right) \mathrm{d} \operatorname{vol}_{g}=0 .
$$

For $n=1$, we obtain $\sigma_{2}=0$, thus, (31) gives zero integral of the "Gaussian P-curvature" of $\mathcal{D}$. Next, for $r=1,2$, (30) reduces to the following:

$$
\begin{array}{r}
\int_{M}\left(3 \sigma_{3}-\operatorname{tr}_{\mathcal{F}}\left(T_{1}\left(A_{N}\right) \mathcal{R}_{N}^{P}+\mathcal{R}_{\mathcal{Z}}^{P}\right)\right) \mathrm{d} \operatorname{vol}_{g}=0 \\
\int_{M}\left(4 \sigma_{4}+\left\langle T_{2}\left(A_{N}\right) \mathcal{Z}, \mathcal{Z}\right\rangle-\operatorname{tr}_{\mathcal{F}}\left(T_{2}\left(A_{N}\right) \mathcal{R}_{N}^{P}+T_{1}\left(A_{N}\right) \mathcal{R}_{\mathcal{Z}}^{P}-\mathcal{R}_{A_{N} \mathcal{Z}}^{P}\right)\right) \mathrm{d}_{\operatorname{vol}_{g}}=0
\end{array}
$$

Example 2. Here is an amazing consequence of (28) and (31). Let us consider a closed connected sub-Riemannian manifold $(M, g ; \mathcal{D}), \operatorname{dim} \mathcal{D} \geq 3$ with a harmonic orthogonal distribution $\mathcal{D}^{\perp}$ and the condition

$$
\operatorname{Ric}^{P} \geq 2 c>0
$$


for some real $c$. Suppose that $(M, g ; \mathcal{D})$ is equipped with a codimension-one foliation $\mathcal{F}$, i.e., $\mathcal{D}=T \mathcal{F} \oplus \operatorname{span}(N)$. Then the image of the function $\sigma_{2}: M \rightarrow \mathbb{R}$ contains an interval $[0, c+\varepsilon]$ for some $\varepsilon>0$.

Indeed, by Reeb type formula (28), $\sigma_{1}(x)=0$ at some $x \in M$. Then $2 \sigma_{2}(x)=-\tau_{2}(x) \leq 0$, see (7). By (31), $\sigma_{2}(\mathcal{F}) \geq c \operatorname{Vol}(M, g)$. Hence, there exists $y \in M$ such that $\sigma_{2}(y)>c$ (otherwise, $\sigma_{2} \leq c$ on $M$, therefore, $\sigma_{2} \equiv c>0$ on $M-$ a contradiction to $\left.\sigma_{2}(x) \leq 0\right)$. Put $\varepsilon=\sigma_{2}(y)-c$. Since $M$ is connected and $\sigma_{2}: M \rightarrow \mathbb{R}$ is a continues function, $\sigma_{2}$ takes all values in the interval $\left[\sigma_{2}(x), \sigma_{2}(y)\right]$, which obviously includes $[0, c+\varepsilon]$.

Using (31), we obtain the following non-existence results for $P$-harmonic, i.e., $\sigma_{1}=0$, and P-totally umbilical, i.e., $A_{N}=\left(\sigma_{1} / n\right) \mathrm{id}_{T \mathcal{F}}$, foliations.

Corollary 2. Let $(M, \mathcal{D}, g)$ be a closed sub-Riemannian manifold with a harmonic distribution $\mathcal{D}^{\perp}$.

(i) If $\operatorname{Ric}^{P}>0$, then there are no P-harmonic codimension-one foliations in $\mathcal{D}$.

(ii) If $\operatorname{Ric}^{P}<0$, then there are no P-totally umbilical codimension-one foliations in $\mathcal{D}$.

Proof. (i) If $\mathcal{F}$ is a $P$-harmonic codimension-one foliation in $\mathcal{D}$, then $2 \sigma_{2}=-\tau_{2} \leq 0$, see (7).

(ii) If $\mathcal{F}$ is a $P$-totally umbilical codimension-one foliation in $\mathcal{D}$, then

$$
2 \sigma_{2}=\frac{n-1}{n}\left(\sigma_{1}\right)^{2} \geq 0
$$

In both cases, (i) and (ii), we obtain a contradiction to integral formula (31).

Obviously, $P$-harmonic and $P$-totally umbilical distributions are harmonic and totally umbilical, respectively, but the opposite is not true.

\section{Some Consequences}

Here, we apply our formulas to sub-Riemannian manifolds with restrictions on the shape operator $A_{N}$ or the induced curvature tensor $R^{P}$.

\subsection{Foliations of Constant Sectional P-Curvature}

The total $r$-th mean curvature of a codimension-one (relative to $\mathcal{D}$ ) foliation $\mathcal{F}$ is given by

$$
\sigma_{r}(\mathcal{F}, N)=\int_{M} \sigma_{r} \mathrm{~d} \operatorname{vol}_{g} .
$$

The following corollary of Theorem 3 generalizes (4), see also Section 4.1 in [4].

Corollary 3. Let $(M, \mathcal{D}, g)$ be a closed sub-Riemannian manifold with $\mathcal{D}=T \mathcal{F} \oplus \operatorname{span}(N)$ and a harmonic orthogonal distribution $\mathcal{D}^{\perp}$. If condition (23) is satisfied, then $\sigma_{r}(\mathcal{F}, N)$ depend on $r, n, c$ and the volume of $(M, g)$ only, i.e., the following integral formula is valid:

$$
\sigma_{r}(\mathcal{F}, N)=\left\{\begin{array}{cc}
c^{r / 2}\left(\begin{array}{c}
n / 2 \\
r / 2
\end{array}\right) \operatorname{Vol}(M, g), & n \text { and } r \text { even, } \\
0, & \text { either } n \text { or } r \text { odd } .
\end{array}\right.
$$

Proof. Using Lemma 3, we obtain

$$
\operatorname{tr}_{\mathcal{F}}\left(T_{r}\left(A_{N}\right) \mathcal{R}_{N}^{P}\right)=\sum_{i=1}^{n}\left\langle R^{P}\left(e_{i}, N\right) N, T_{r}\left(A_{N}\right) e_{i}\right\rangle=c \operatorname{tr}_{\mathcal{F}} T_{r}\left(A_{N}\right)=c(n-r) \sigma_{r} .
$$

From (30) we obtain the equality

$$
(r+2) \sigma_{r+2}(\mathcal{F}, N)=c(n-r) \sigma_{r}(\mathcal{F}, N) .
$$


Since $\sigma_{1}(\mathcal{F}, N)=0$, see (28), by induction we get $\sigma_{r}(\mathcal{F}, N)=0$ for any odd $r$. For even $r=2 s$ and $n=2 l$, using (33) and induction, we obtain $\sigma_{2 s}(\mathcal{F}, N)=c^{s}\left(\begin{array}{l}l \\ s\end{array}\right) \operatorname{Vol}(M, g)$.

\subsection{P-Totally Umbilical Foliations}

Here, we obtain similar to (32) integral formulas when $(M, \mathcal{D}, g)$ has the property

$$
\operatorname{Ric}_{X, N}^{P}=C\langle X, N\rangle, \quad X \in \mathcal{D},
$$

for some $C \in \mathbb{R}$ and $\mathcal{F}$ (with $\operatorname{dim} \mathcal{F}>1$ ) is a $P$-totally umbilical foliation. Please note that Einstein manifolds satisfy condition (34) when $\mathcal{D}=T M$.

The following corollary of Theorem 3 generalizes result in ([4] Section 4.2) on codimensionone totally umbilical foliations of Einstein manifolds.

Corollary 4. Let $(M, \mathcal{D}, g)$ be a closed sub-Riemannian manifold with $\mathcal{D}=T \mathcal{F} \oplus \operatorname{span}(N)$, $P$-totally umbilical foliation $\mathcal{F}$ with $\operatorname{dim} \mathcal{F}>1$, a harmonic orthogonal distribution $\mathcal{D}^{\perp}$, and satisfying (34). Then $\sigma_{r}(\mathcal{F}, N)$ depends on $r, n, C$ and the volume of $(M, g)$ only, i.e., the following formula holds:

$$
\sigma_{r}(\mathcal{F}, N)=\left\{\begin{array}{cc}
(C / n)^{r / 2}\left(\begin{array}{c}
n / 2 \\
r / 2
\end{array}\right) \operatorname{Vol}(M, g), & n, r \text { even }, \\
0, & r \text { odd } .
\end{array}\right.
$$

Proof. In this case, $T_{r}\left(A_{N}\right)$ has the form

$$
T_{r}\left(A_{N}\right)=a_{r}\left(\sigma_{1} / n\right)^{r} \operatorname{id}_{T \mathcal{F}}, \quad \text { where } \quad a_{r}=\sum_{i=0}^{r}(-1)^{r-i}\left(\begin{array}{c}
n \\
i
\end{array}\right)
$$

Since $\operatorname{tr}_{\mathcal{F}} T_{r}\left(A_{N}\right)=(n-r) \sigma_{r}=(n-r)\left(\begin{array}{l}n \\ r\end{array}\right)\left(\sigma_{1} / n\right)^{r}$, then

$$
a_{r}=\frac{n-r}{n}\left(\begin{array}{l}
n \\
r
\end{array}\right), \quad T_{r}\left(A_{N}\right)=\frac{n-r}{n} \sigma_{r} \operatorname{id}_{T \mathcal{F}} .
$$

By our assumption (34),

$$
\operatorname{Ric}_{\mathcal{Z}, N}^{P}=0, \quad \operatorname{Ric}_{N, N}^{P}=C .
$$

Thus, for a closed manifold with a P-totally umbilical foliation $\mathcal{F}$ and condition (34), formula (30) becomes similar to (33):

$$
\sigma_{r+2}(\mathcal{F}, N)=\frac{C}{n} \cdot \frac{n-r}{r+2} \sigma_{r}(\mathcal{F}, N) .
$$

Using induction similarly to Corollary 3 , we obtain (35).

Remark 2. Our integral formulas provide more conditions for the mean curvature $H=\sigma_{1} / n$ of $\mathcal{F}$. In the case of a P-totally umbilical codimension-one foliation in $\mathcal{D}$, such conditions can be easily derived from (30) using $\sigma_{r}=\left(\begin{array}{c}n \\ r\end{array}\right) H^{r}$ :

$$
\begin{aligned}
& \int_{M} H^{r-1}\left(H^{3}(n-1)(n-r-1) n\right. \\
& \left.-H(n-1)(r+1) \operatorname{Ric}_{N, N}^{P}-r(r+1) \operatorname{Ric}_{\mathcal{Z}, N}^{P}\right) \mathrm{d} \mathrm{vol}_{g}=0 .
\end{aligned}
$$

Here we used the following identity with binomial coefficients:

$$
\sum_{j=1}^{r}(-1)^{j-1} \frac{n-r+j}{n}\left(\begin{array}{c}
n \\
r-j
\end{array}\right)=\left(\begin{array}{c}
n-2 \\
r-1
\end{array}\right) .
$$


These integrals contain polynomials depending on $H$, and one can obtain obstructions for existence of P-totally umbilical foliations. For example, if $r=n-1$, then (36) reads as

$$
\int_{M} H^{n-2}\left(H \operatorname{Ric}_{N, N}^{P}+\operatorname{Ric}_{\mathcal{Z}, N}^{P}\right) \mathrm{d}^{\operatorname{vol}_{g}}=0 ;
$$

thus, if $\operatorname{Ric}^{P}>0$, then any P-totally umbilical codimension-one foliation in $\mathcal{D}$ with $\mathcal{Z}=0$ is P-totally geodesic, i.e., $A_{N}=0$.

\section{Conclusions}

In the article, integral formula (2) and its consequences for foliated space forms are generalized for a Riemannian manifold equipped with a foliation $\mathcal{F}$ and a unit vector field $N$ orthogonal to $\mathcal{F}$. The results can be applied to foliated sub-Riemannian manifolds. Moreover, our integral formulas can be easily extended for non-integrable distributions and foliations defined outside of a "singularity set" $\Sigma$ (a finite union of pairwise disjoint closed submanifolds of codimension at least $k$ of a closed manifold $M$ ) under additional assumption of convergence of certain integrals. Namely, instead of the Divergence theorem, we apply the following result, see [9]: if $(k-1)(q-1) \geq 1$ and $X$ is a vector field on $M \backslash \Sigma$ such that $\int_{M}\|X\|^{q} \mathrm{~d} \mathrm{vol}_{g}<\infty$, then $\int_{M} \operatorname{div} X \mathrm{~d} \operatorname{vol}_{g}=0$. One can also try to extend our integral formulas for holomorphic foliations of complex (sub-)Riemannian manifolds, see [15] for the case of Riemannian manifolds, i.e., $\mathcal{D}=T M$, and for foliations of metric affine manifolds, see [16]. Finally note that our integral formulas are less cumbersome than the integral formulas in [17].

Funding: This research received no external funding.

Conflicts of Interest: The author declares no conflict of interest.

\section{References}

1. Reeb, G. Sur certaines propriétés topologiques des variétés feuilletées, Actualités. Sci. Ind. 1952, 1183, 144.

2. Candel, A.; Conlon, L. Foliations, I; Graduate Studies in Mathematics; AMS: Providence, RI, USA, $2000 ;$ Volume 23.

3. Andrzejewski, K.; Rovenski, V.; Walczak, P. Integral formulas in foliations theory. In Geometry and Its Applications; Springer Proceedings in Mathematics \& Statistics; Springer: Cham, Switzerland, 2014; Volume 72, pp. 73-82

4. Andrzejewski, K.; Walczak, P. The Newton transformation and new integral formulae for foliated manifolds. Ann. Glob. Anal. Geom. 2010, 37, 103-111. [CrossRef]

5. Bejancu, A.; Farran, H. Foliations and Geometric Structures; Springer: Dordrecht, The Netherlands, 2006.

6. Brito, F.; Langevin, R.; Rosenberg, H. Intégrales de courbure sur des variétés feuilletées. J. Diff. Geom. 1981, 16, 19-50. [CrossRef]

7. Rovenski, V.; Walczak, P. Topics in Extrinsic Geometry of Codimension-One Foliations; Springer Briefs in Mathematics; Springer: New York, NY, USA, 2011.

8. Rovenski, V.; Walczak, P. Extrinsic Geometry of Foliations; Birkhäuser: Basel, Switzerland, 2021.

9. Walczak, P. Integral formulae for foliations with singularities. Coll. Math. 2017, 150, 141-148. [CrossRef]

10. Reeb, G. Sur la courboure moyenne des variétés intégrales d'une équation de Pfaff $\omega=0$. C. R. Acad. Sci. Paris 1950, 231, 101-102.

11. Nora, T. Seconde Forme Fondamentale d'une Application et d'un Feuilletage. Ph.D. Thesis, Université de Limoges, Limoges, France, 1983; 115p.

12. Gray, A. Pseudo-Riemannian almost product manifolds and submersions. J. Math. Mech. 1967, 16, 715-737.

13. Jost, J. Riemannian Geometry and Geometric Analysis, 7th ed.; Springer: Cham, Switzerland, 2017.

14. Sullivan, D. A homological characterization of foliations consisting of minimal surfaces. Comment. Math. Helv. 1979, 54, $218-223$. [CrossRef]

15. Svensson, M. Holomorphic foliations, harmonic morphisms and the Walczak formula. J. Lond. Math. Soc. 2003, 68, 781-794. [CrossRef]

16. Rovenski, V. Integral formulas for a metric-affine manifold with two complementary orthogonal distributions. Glob. J. Adv. Res. Class. Mod. Geom. 2017, 6, 7-19.

17. Rovenski, V. Integral formulas for a Riemannian manifold with two orthogonal distributions. Cent. Eur. J. Math. 2011, 9, 558-577. [CrossRef] 